\title{
Design and Development of ZnO Based Gas Sensor for Fruit Ripening Detection
}

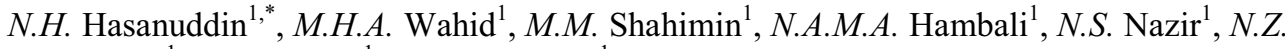 \\ Khairuddin ${ }^{1}$, M.M. Ramli ${ }^{1}$, and S.S.M. Isa ${ }^{1}$. \\ ${ }^{1}$ Semiconductor Photonics \& Integrated Lightwave Systems (SPILS), School of Microelectronic \\ Engineering, Universiti Malaysia Perlis, Pauh Putra Campus, Malaysia.
}

\begin{abstract}
This work focuses on the nondestructive fruit ripeness detection system at different maturity stages. The study involved a computational approach whereby the detector is simulated and characterized. The development of the 2 dimensional finite elements provides deeper understanding in an isotropic media of the sensor. The paper presents a sensitive layer of the zinc oxide with a thickness of $0.5 \mu \mathrm{m}$ on $\mathrm{LiNbO3}$ piezoelectric substrate for ethylene $(\mathrm{C} 2 \mathrm{H} 4)$ gas sensing application in order to distinguish maturity level of fruits. In addition the sensor acts as a better device for sensing the amount of ethylene gases that releases from mature fruits.
\end{abstract}

\section{Introduction}

Sensors are part of our daily life and have been applied in variety of fields and gas sensing is only one of the uses. Theoretically surface acoustic wave sensors (SAWs) categorized in microelectromechanical systems (MEMS) which depend on the modulation of acoustic surface to detect a physical phenomenon. Microelectromechanical systems (MEMS) are one of the areas that are widely used for gas sensing purpose, because of the advantages it brings in efficiency, robustness and cost effectiveness [1,2]. By introducing ethylene gaseous as an input to the sensors, receiver will receives and transduces input into a mechanical wave [1]. The sensor then transduces again the mechanical wave into electrical signal. Ethylene $\left(\mathrm{C}_{2} \mathrm{H}_{4}\right)$ is fruit ripening agent. $\mathrm{C}_{2} \mathrm{H}_{4}$ gas plays an important role in the ripening process of climacteric fruits. Ethylene molecule composed from two carbon atoms bond with four hydrogen atoms. Knowing $\mathrm{C}_{2} \mathrm{H}_{4}$ gaseous concentration that been released by fruits, may distinguish the maturity level and remaining shelf life can be predicted [3].

In this research, the eigen frequency of SAW gas detector is investigated. Surface acoustic wave gas detector is based on the development of acoustic energy, where the transferring of energy is more responsive near to the surface of the detector [4]. SAW gas sensor consists of interdigitated transducers (IDT) as shown in Figure 1 which are placed on the top of substrate in order to transmit and receive acoustic waves. The IDTs layer is divided into two sets which is called the transmitter and the second set the receiver. Transmitter converts electrical signal into acoustic wave that travels along the piezoelectric

\footnotetext{
* Corresponding author: irhafika@gmail.com
} 
substrate, then receiver converts back into electrical signal for detection and analysis purposes $[5,6]$.

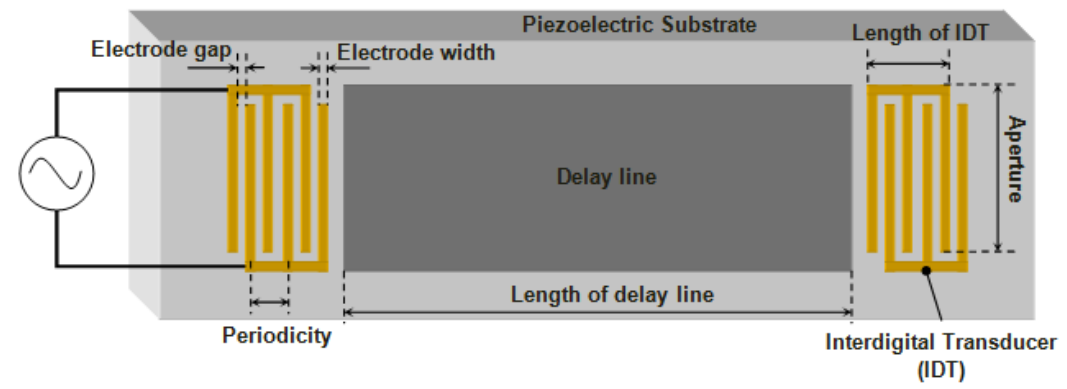

Fig. 1. Surface Acoustic Wave Sensor [1].

\section{Material selection}

\subsection{Piezoelectric substrate material}

Piezoelectric material is chosen based on the electrochemical coefficient $\left(\mathrm{K}^{2}\right)$, polarization and orientation. The extension of the wave interacts between the energy conversion that will effect in efficient measurement or $\left(\mathrm{K}^{2}\right)$ which insisted by $\mathrm{K}^{2}=2\left(\mathrm{~V}_{\mathrm{f}}-\mathrm{V}_{\mathrm{m}}\right) / \mathrm{Vf}$ where $\mathrm{Vf}$ and $\mathrm{Vm}$ represent free surface and metalized surface phase velocities [7]. Most popular substrate materials are Lithium Niobate $\left(\mathrm{LiNbO}_{3}\right)$ and Lithium Tantalate $\left(\mathrm{LiTaO}_{3}\right)$ because of high $\mathrm{K}^{2}$. Mode of SAW need to be considered before choosing between these two common substrate materials, because it has different acoustic properties in which each of them will provide the differences criteria with conductivity based sensitivity. In this study, $\mathrm{XY} \mathrm{LiNbO}_{3}$ is selected because its advantage that can support a single mode SAW in three directions $\mathrm{U}_{\mathrm{x}}, \mathrm{U}_{\mathrm{y}}$, and $\mathrm{U}_{\mathrm{z}} \cdot[6]$.

\subsection{Interdigital transducers (IDTs) material}

Aluminum has been selected as IDTs material because it is easy to deposit and cohere well with common oxide substrate compared to gold ( $\mathrm{Au}$ ) and chromium $(\mathrm{Cr})$. Basically two set of IDTs commonly used in acoustic wave device, which are input and transmitter. Input work in generating energy while transmitter will convert electrical energy into mechanical energy. In the meantime output IDTs work as receiver that converts back mechanical energy into electrical energy in which at this stage occurrence of detection and analysis of ethylene gas [7].

\subsection{Intermediate layer material}

$\mathrm{ZnO}$ is chosen as intermediate layer because intermediate layer material should be dielectric layer in order to interact with piezoelectric substrate material. $\mathrm{ZnO}$ has low acoustic velocity about $2531 \mathrm{~m} / \mathrm{s}$ compared to other dielectric material velocities. By varying the thickness of the $\mathrm{ZnO}$ intermediate layer it may increase effectiveness towards rate of gas absorption [5]. 


\subsection{Sensing layer material}

Sensing material is very important to make sure that the absorption occurred. $\mathrm{ZnO}$ nanowire is much useful in sensor application due to its good conductivity compared to others. Deformation occurs on $\mathrm{ZnO}$ due to the absorption of ethylene gas molecules on the nanowire which lead to alterations in conductivity [5].

\section{Multiphysics modelling}

Figure 2 shows a 2D schematic layer of the SAW zinc oxide based gas sensor for sensing ethylene gas that releases from fruits. The dimension of $\mathrm{LiNbO}_{3}$ substrate is $30 \mu \mathrm{m}$ in the $\mathrm{x}-$ axis and $4 \mu \mathrm{m}$ in the $\mathrm{z}$-axis. The dimension is based on the limitation of generated nodes number. Intermediate layer of $\mathrm{ZnO}$ with $\mathrm{x}$-axis $30 \mu \mathrm{m}$ and $\mathrm{z}$-axis $0.2 \mu \mathrm{m}$ is placed on the $\mathrm{LiNbO}_{3}$ substrate. The $\mathrm{ZnO}$ sensing layer is placed on top of all substrates with $30 \mu \mathrm{m}$ in length and $0.5 \mu \mathrm{m}$ in height. The IDTs aluminum substrate is given by $1 \mu \mathrm{m}$ as the width and $0.1 \mu \mathrm{m}$ as the height, and it is defined massless electrodes in order to ignore the second order effects of the electrodes [8].

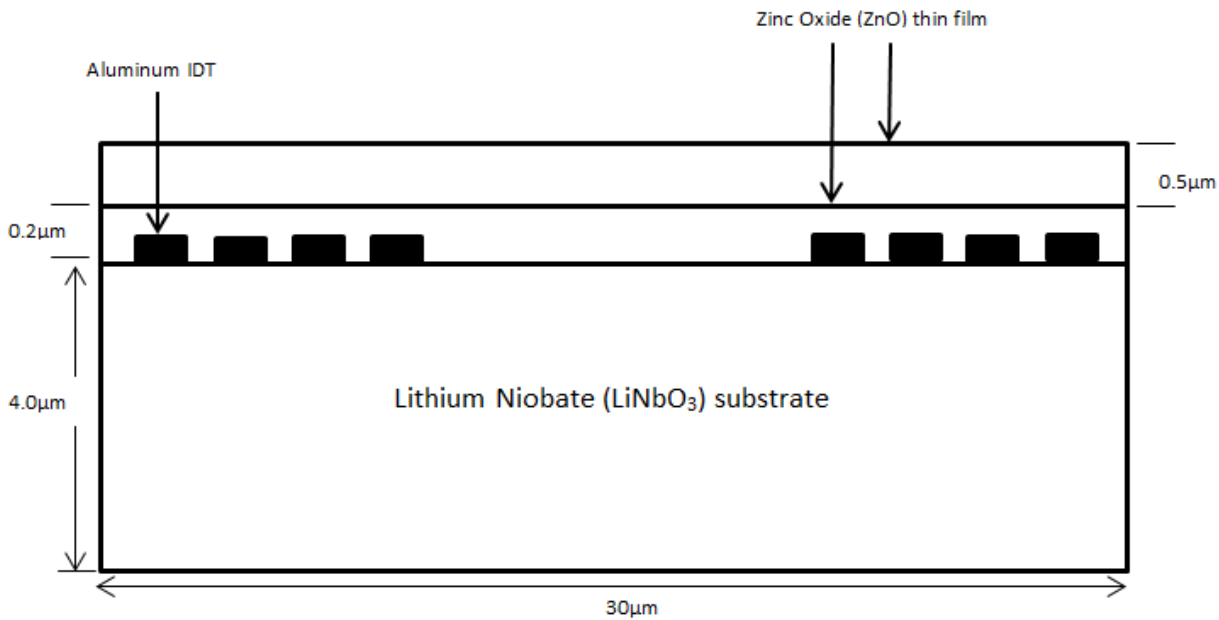

Fig. 2. SAW Sensor as the Sensing Material.

The simulations are executed using transient analysis for a simulation time of $0.1 \mathrm{~s}$ with 0.001 time step. Initially all the exterior condition of mechanical boundaries are set to be free exclude for the third boundary that is set to be fixed. All exterior electrical boundaries condition is fixed to be zero charge and those for transmit aluminium IDTs, electrode 1 and electrode 3 are positioned to be in positive electrical potential and zero potential is set to the second and fourth electrode. This combination of electrical boundary conditions corresponds to an open circuit configuration, which is typically suitable for sensing applications [9]. The use of periodic boundary condition implies that the frequencies of interest correspond to wavelengths that are integer fractions of the width of the geometry. The lowest SAW eigenmode has its wavelength equal to the width of the geometry, that is, $4 \mu \mathrm{m}$. Using this along with the Rayleigh wave velocity for the given piezoelectric substrate material, an estimation of the resonance frequency of interest can be established. The information can be used in the eigenfrequency solver, which helps it to find out the resonance frequencies close to this estimated number. 
The wavelength of the substrate is described by equation 1 and the center frequency is described by equation $2[10,11,12]$;

$$
\begin{gathered}
\lambda=2\left(W_{e}+W_{s p}\right) \\
f_{0=} \frac{V 0}{\lambda}
\end{gathered}
$$

where $\lambda$ is the wavelength of acoustic wave, $W_{\mathrm{e}}$, width of each electrode and $\mathrm{W}_{\mathrm{sp}}$ is the space between each electrode. The operating frequency, $f_{0}$, is determined by the propagation velocity, $V_{0}$ and the wavelength, $\lambda$. A yz-cut $\mathrm{LiNbO}_{3}$ whose Rayleigh wave velocity (vR) is around $3488 \mathrm{~m} / \mathrm{s}$. This gives an estimate of the lowest SAW frequency $\left(\mathrm{f}_{0}\right)$ to be $872 \mathrm{MHz}$.

Figure 3 shows the meshes cross section model. The maximum node size is chosen to be smaller than the wavelength to ensure the compensation of appropriate harmonics. This resulted in 3873 elements in the mesh. Meshing process is performed to determine the nodes in SAW gas sensor detecting structure which can be seen that the highest density of the nodes is directly under IDTs location.

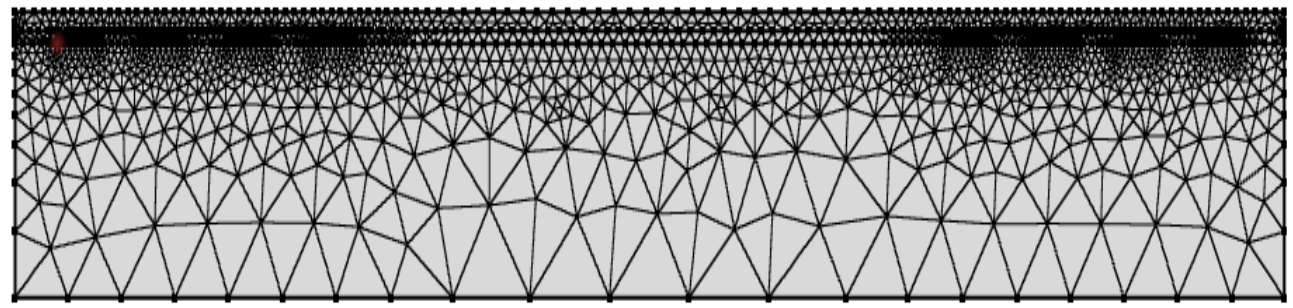

Fig. 3. Meshed image of 2D cross-section of the SAW Gas Sensor.

\section{Results and discussion}

Figure 4 illustrates the difference color variation due to the applied voltage on the input IDTs. It is shown that more energy at the surface rather than bulk substrate. This proves that SAW gas sensor confines more energy at the surface of piezoelectric substrate [12].

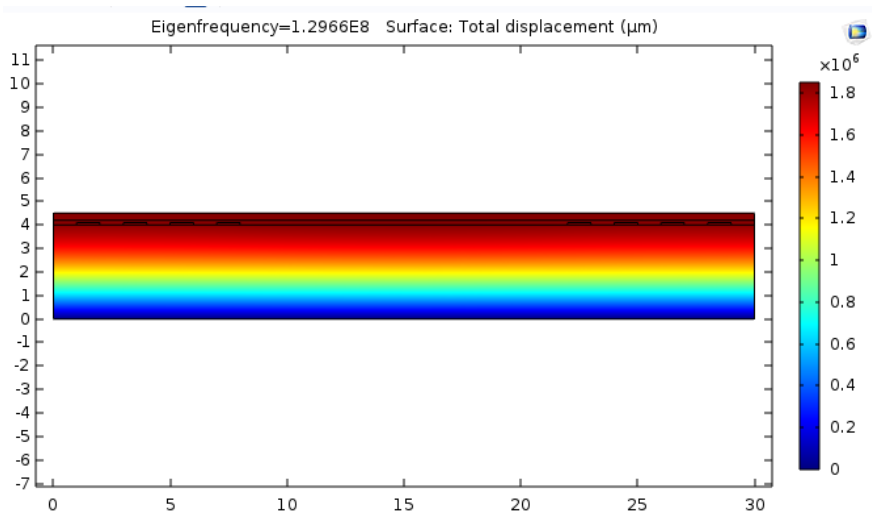

Fig.4. SAW wave propagation with the absence of $\mathrm{C}_{2} \mathrm{H}_{4}$ gas. 
Frequency dependent study is performed and produced its output as depicted in Figure 5. The acoustic wave can be observed, at the determined frequency, traveling at the top of substrate.

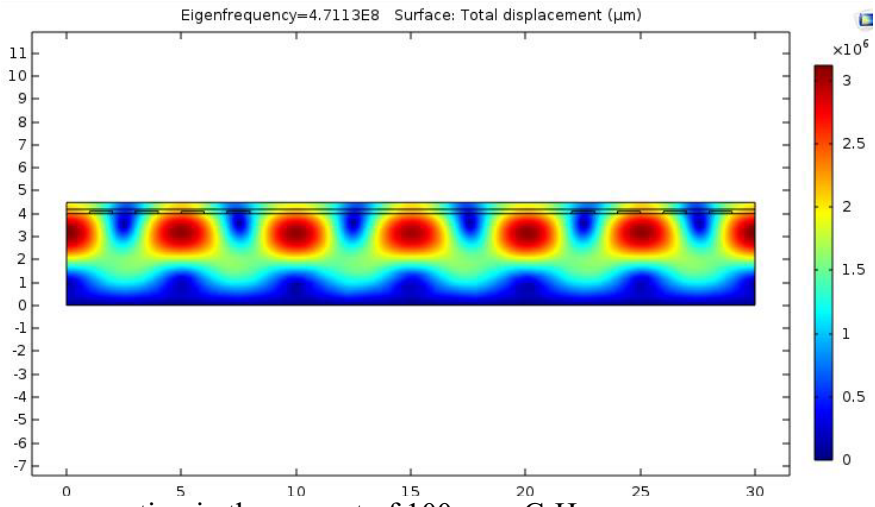

Fig. 5. SAW wave propagation in the present of $100 \mathrm{ppm} \mathrm{C}_{2} \mathrm{H}_{4}$ gas.

A parameter sweep of the electrode resonant frequency or eigenfrequency is done during frequency dependent study. This is done by firstly sweeping the parameter from 1.0E8 to 7.0E8 as displayed in Figure 6.

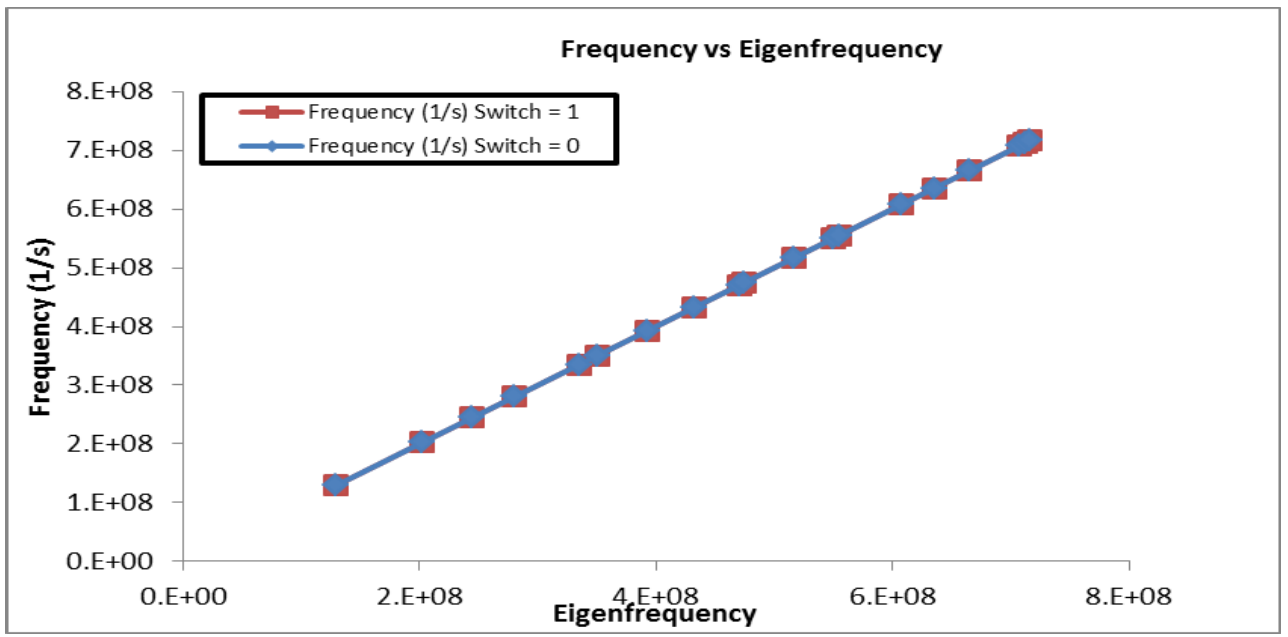

Fig. 6. Eigenfrequency sweep showing frequency(1/s) traveling along the substrate surface.

Figure 6 shows the exposure of $\mathrm{C}_{2} \mathrm{H}_{4}$ to the $\mathrm{SAW}$ gas detector that engaged a $2 \mathrm{KHz}$ frequency shift. The frequency shift presents the existence of $\mathrm{C}_{2} \mathrm{H}_{4}$ gas releasing from fruit.

\section{Conclusions}

A simulation of SAW ethylene gas detector with $\mathrm{ZnO}$ as core material is successfully developed. An optimum frequency for this ethylene detector to be operated is $872 \mathrm{MHz}$. The $\mathrm{x}-\mathrm{y} \mathrm{LiNbO}_{3}$ substrate is used as a piezoelectric layer as it can support a single mode SAW in three directions $U_{x}, U_{y}$, and $U_{z}$. The simulation is prepared in order to investigate the nature of the gas detector in the existence of ethylene gaseous. The outcome from the simulation shows that the total displacement at a determined frequency, gives a slightly 
changes and this concludes that with the present of $\mathrm{C}_{2} \mathrm{H}_{4}$ exposed to the surface of gas detector, frequency is shifted by about $200 \mathrm{~Hz}$. Future work includes the simulation of the full sensor for ripening identification as well as the fabrication of SAW gas sensor.

\section{References}

1. D.S. Ballantine, Acoustic Wave Sensors: Theory, Design, \& Physico-Chemical Applications (Academic Press,1997)

2. Morgan, P. David, IEEE International Frequency Control Symposium (1998)

3. S. Janssen, K. Schmitt, M. Blanke, M.L. Bauersfeld, J. Wöllenstein, W. Lang, Mathematical, Physical and Engineering Sciences, 372, 20130311 (2017)

4. Y. Rao, G. Zhang, Proceedings of the COMSOL Conference, Boston (2007)

5. Hercules G du Plessis, Proceedings of the COMSOL Conference in Rotterdam (2013)

6. H. Wohltjen, R. Dessy, Analytical Chemistry, 51, 1465 (1979)

7. Nurul Izzati Binti Mohamad Isa, Modelling of Intermediate Layer for Love Mode SAW device thesis, Universiti Teknologi Malaysia, Johor (2011)

8. L.J. Bie, X.N. Yan, Y. Jing, Y.Q. Duan, Z.H. Yuan, Sensors and Actuators B: Chemical, 126, 604 (2007)

9. M.Z. Atashbar, A.Z. Sadek, W. Wlodarski, S. Sriram, M. Bhaskaran, C.J. Cheng, R.B. Kaner, K. Kalantar Zadeh, Sensors and Actuators B: Chemical, 138, 85 (2009)

10. K. Ho, E. R. Lindgren, K. S. Rawlinson, L. K. McGrath, J. L. Wright, Sensors, 3, 236 (2003)

11. S. Ahmadi, F. Hassani, C. Korman, M. Rahaman, M. Zaghloul, Sensors, 3, 1129 (2004)

12. Di Francia, Girolamo, Alfano, Brigida, La Ferrara, Vera, Journal of Sensors (2009) 